\title{
Plasma metabolites in first episode psychosis: target and untarget mass-spectrometer-based analysis
}

\begin{abstract}
Alana Costa, Helena Joaquim, Leda Talib, Marcus Zanetti, Frederik Dethloff, Chistoph Turck, Wagner Gattaz
\end{abstract} alanacosta@usp.br

\section{Introduction}

The development of sensitive and accurate biomarkers is highly required for schizophrenia (SCZ) and bipolar disorder (BD). Lipids play an increasingly recognized role in the neuronal function and plasticity of the brain. Since lipid metabolism is altered differently in psychiatric diseases, alterations in the membrane lipid profile can allow discrimination between subjects in first episode psychosis, thus suggesting a specificity of the findings for these diseases.

\section{Objective}

- To quantify the plasmatic metabolites of subjects in first episode psychosis and healthy controls

- To define a set of metabolites that may aid in the clinical differentiation.

\section{Material and Methods}

\section{Subjects}

Table 1. Socio-demographic characteristics and clinical assessment

\begin{tabular}{lcccc}
\hline \multicolumn{1}{c}{ Diagnosis } & $\begin{array}{c}\text { SCZ } \\
(n=28)\end{array}$ & $\begin{array}{c}\text { BD } \\
(n=27)\end{array}$ & $\begin{array}{c}\text { Controls } \\
(n=30)\end{array}$ & $p$ \\
\hline Gender (M/F) & $17 / 11$ & $5 / 22$ & $16 / 14$ & $\mathbf{0 . 0 3}$ \\
Age (mean \pm sd) & $26.0 \pm 7.4$ & $28.9 \pm 5.6$ & $26.2 \pm 3.9$ & 0.12 \\
Education (mean $\pm \mathrm{sd})$ & $10.8 \pm 3.5$ & $13.7 \pm 2.1$ & $14.7 \pm 2.3$ & $\mathbf{0 . 0 1}$ \\
PANSS (mean $\pm \mathrm{sd}$ ) & $78 \pm 22$ & - & - & \\
HAM-D (mean $\pm \mathrm{sd})$ & - & $15 \pm 8$ & - & \\
YMRS (mean $\pm \mathrm{sd}$ ) & - & $9 \pm 8$ & - &
\end{tabular}

M male; F female; sd standard deviation; SCZ Schizophrenia; BD Bipolar Disorder; PANSS Positive and Negative Syndrome Scale; HAM-D Hamilton Depression Scale; YMRS Young Mania Rating Scale; $\mathbf{p}$ significance of Chi-square test.

\section{Metabolites quantification}

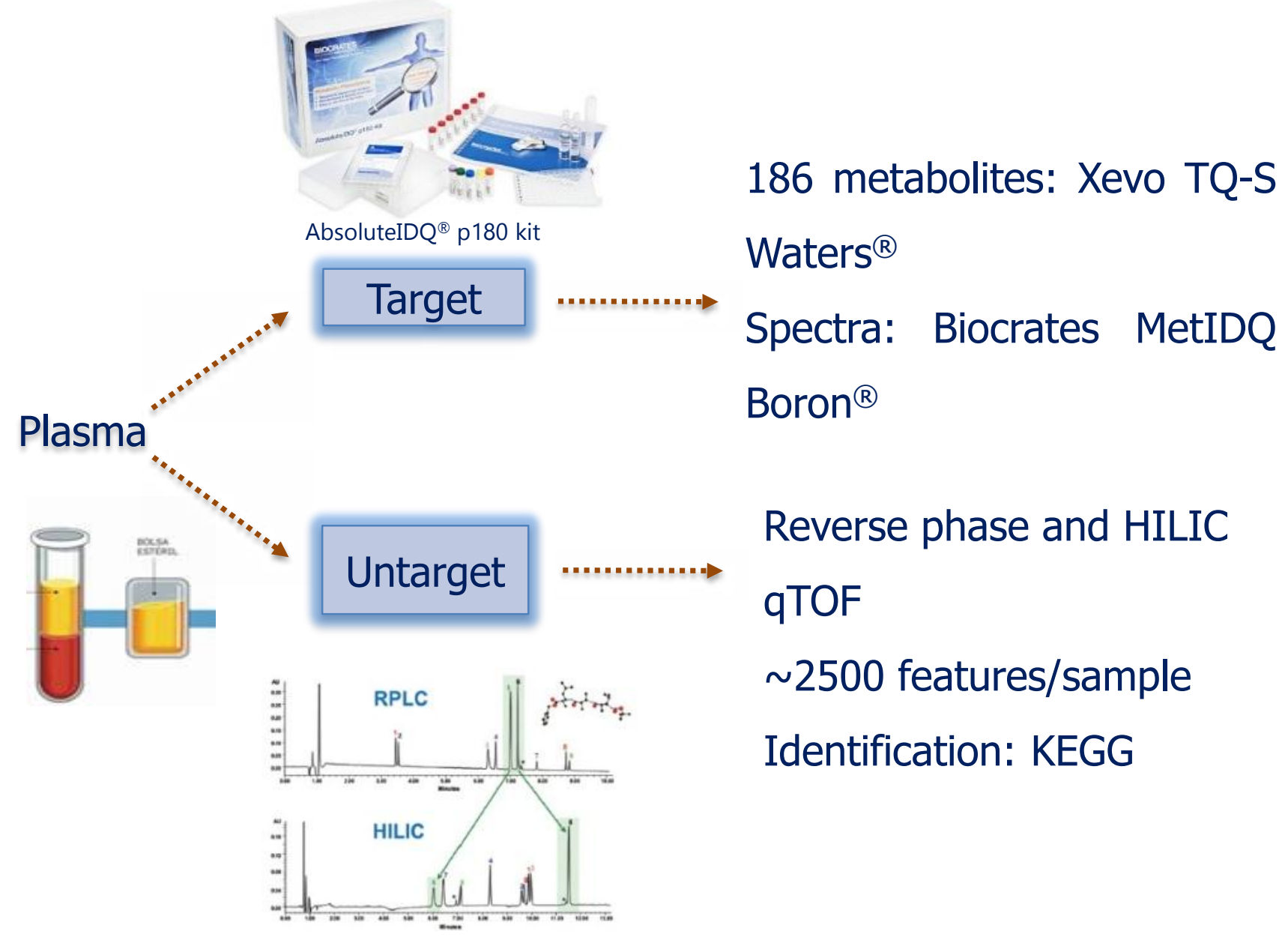

\section{Results}

\begin{tabular}{|c|c|c|c|}
\hline \multirow{2}{*}{ Target } & \multicolumn{2}{|r|}{ SCZ } & $B D$ \\
\hline & Acylcarnitines & $\square$ & ש \\
\hline & Phospholipids & $\boldsymbol{\uparrow}$ & 个1 \\
\hline & Lysophospholipids & $\boldsymbol{\uparrow}$ & $\uparrow$ \\
\hline & Sphingolipids & $\eta$ & $\uparrow$ \\
\hline
\end{tabular}

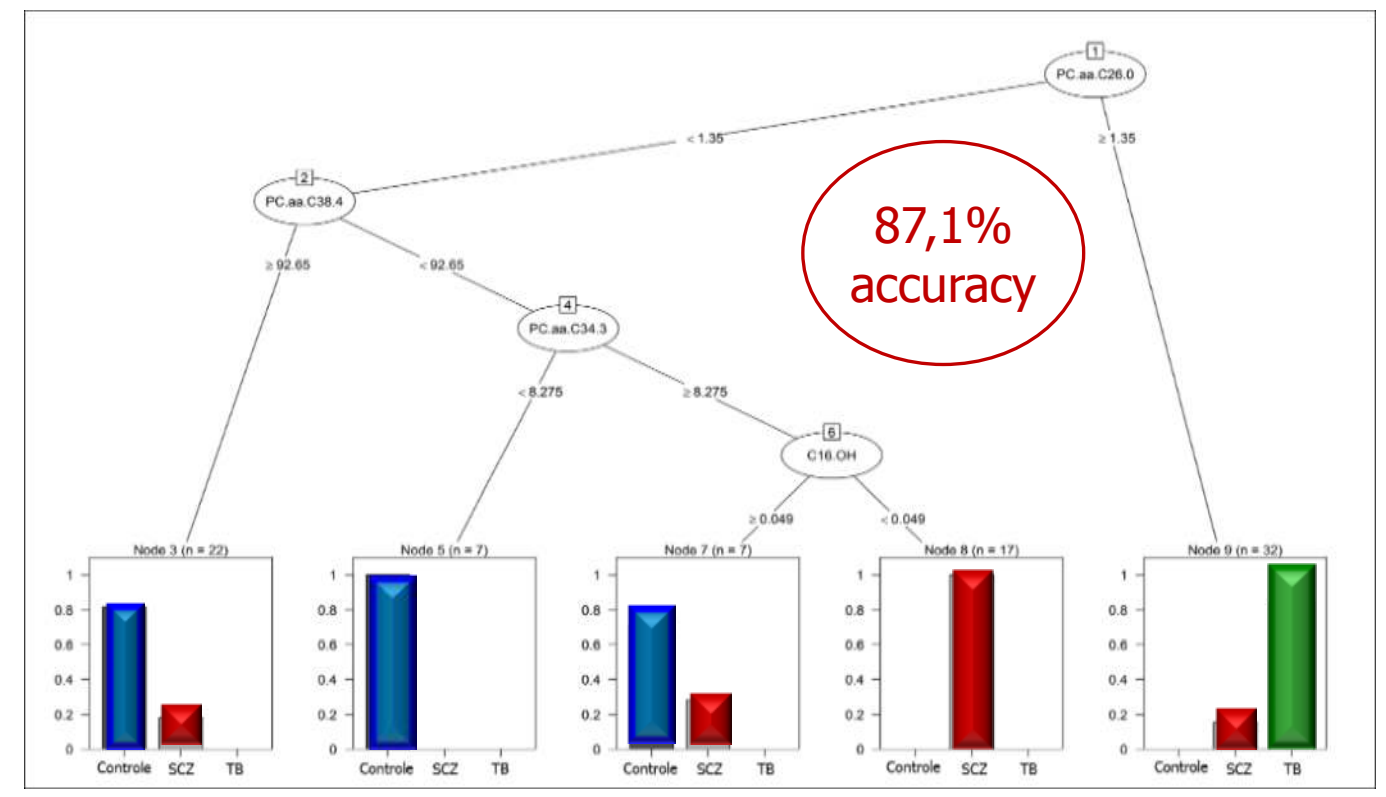

Figure 1. Classification And Regression Tree model for plasmatic metabolites

Untarget Preliminary analyzes of $\sim 2500$ metabolites using unsupervised exploratory multivariate analyzes.

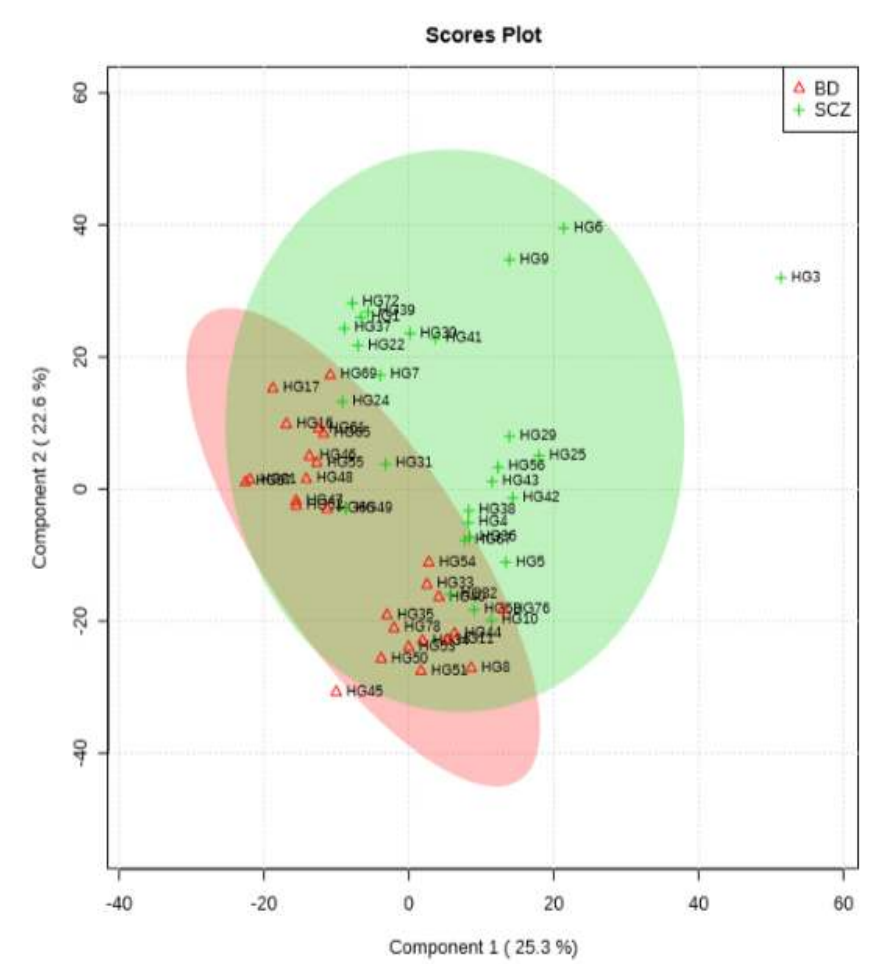

Figure 2: PLS-DA analysis

\section{Conclusions}

- Target analysis provided the separation of SCZ and BD patients with an accuracy of $87 \%$ based on 4 metabolites.

- Untarget analysis could differentiate subjects in two subgroups biologically more homogeneous.

- These subgroups comprised both SCZ and BD patients, suggesting a biological homogeneity in both diagnoses groups regarding the metabolites investigated.

- Further studies should clarify the clinical relevance of these findings for the subgrouping of psychoses

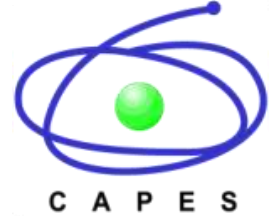

